\title{
A New Required Reserve Capacity Determining Scheme with Regard to Real time Load Imbalance
}

\author{
Joon Hyung Park*, Sun Kyo Kim** and Yong Tae Yoon ${ }^{\dagger}$
}

\begin{abstract}
Determination of the required reserve capacity has an important function in operation of power system and it is calculated based on the largest loss of supply. However, conventional method cannot be applied in future power system, because potential grid-connected distributed generator and abnormal temperature cause the large load imbalance. Therefore this paper address new framework for determining the optimal required reserve capacity taking into account the real time load imbalance. At first, we introduce the way of operating reserve resources which are the secondary, tertiary, Direct Load Control (DLC) and Load shedding reserves to make up the load imbalance. Then, the formulated problem can be solved by the Probabilistic Dynamic Programming (PDP) method. In case study, we divide two cases for comparing the cost function between the conventional method and the proposed method.
\end{abstract}

Keywords: Operating reserve, Required reserve capacity, Secondary reserve, Tertiary reserve, Direct load control, Load imbalance, Probabilistic dynamic programming

\section{Introduction}

Determination of the required reserve capacity has an important function in a power system aiming to reach reliable power system operation which meets the load demand. Therefore, in power systems, it is essential to keep a certain amount of operating reserves capacity because of clearing load imbalance due to generator outages and load fluctuations.

There are many researches which study calculating operating reserves capacity. Most of researches determine the amount of operating reserves capacity by considering the loss of largest generator and a certain percentage of the peak load. [1] For example, Union for the Coordination of Transmission of Electricity (UCTE) merely recommends that the sum of secondary and tertiary control reserves that is operating reserves should cover the largest loss of supply. Moreover, in the areas of UCTE, the required amount of primary control reserves is clearly calculated, however the rule for determining secondary and tertiary control reserves capacity is not precisely specified. [2]

This method is easy to define the amount of operating reserves capacity for System Operator (SO), however, it is not considered the economic and proper reliability aspects of operations since they do not account for random events affecting load and generation balance and in real time and market variables such as reserves price. [3, 4] As a result,

$\dagger$ Corresponding Author: Dept. of Electrical and Computer Engineering, Seoul National University, Korea. (ytyoon@snu.ac.kr)

* Dept. of Electrical and Computer Engineering, Seoul National

University, Korea. (cdove@snu.ac.kr)

** KEPCO Economy and Management Research Institute, Korea (kimsunkyo@kepco.co.kr)

Received: April 14, 2014; Accepted: November 4, 2014 although this method is applicable to the past power system with low volatility load, it cannot implement to power system of the future, smart-grid.

The current methods that determine the amount of operating reserves capacity cannot be applied in the smartgrid, future power system, because of the reliability of the power system and the economics of power system. Prior to discuss how to determine the amount of operating reserves capacity we want to argue the characteristic of future power system. [5] Grid-connected distributed generator, wind power, solar power introduce power fluctuations on the grid, because their power output is influenced by the climatic. Their power fluctuations on the grid leads to large demand volatility, therefore it is not reasonable to decide operating reserves at fixed value. $[6,7]$ Moreover, abnormal temperature cause the imbalance of demand in now and future power system. For these reasons, SO should be determined to apply real time response operating reserves capacity rather than fixed operating reserves capacity. For applying real time response operating reserves capacity, it is necessary to have various reserves resources which respond to large demand volatility, and real-time demand information.

In future power system, there is a variety of operating reserves resources which is energy storage system (ESS) and direct load control (DLC). [8-10] These resources are able to respond to load imbalance directly for satisfying power system reliability. Therefore, SO could decide the amount of operating reserves capacity every fifteen minutes by applying the real-time demand information.

This paper addresses the issue of how to determine the amount of reserves capacity by real time in future power system, smart-grid. The operating reserves are divided into 
secondary control reserve, tertiary control reserve and DLC reserve. We will focus on the subjects of determination of secondary control reserves capacity with consideration of tertiary control reserve and DLC reserve, which is supervisory with respect to the secondary control to replace secondary control reserves. In order to determine the amount of secondary, tertiary control and DLC reserves capacity, we use Probabilistic Dynamic Programming (PDP) to solve problems in which the current period's cost or the nest period's state are random. [12] In addition, for applying the real time load imbalance, we take probabilistic load imbalance model that the uncertainty of demand is represented by fluctuation probabilistic. We assume that the cost of buying reserves in the market can be assessed by a linear function representing the bids offered by the market agents for selling upward and downward reserves.

The remainder of this paper is organized as follows. The paper begins with classification and definition of operating reserves, Section II. Section III presents the required new approach to determine the amount operating reserve capacity. Section IV introduces the mathematical modeling of proposed scheme and then it is applied to a study case in Section V. Finally, Section V contains some relevant conclusions.

\section{Classification and Definition of Operating Reserves}

Operating reserves is defined as the active power capacity that can be deployed to assist with generation and load balance and frequency control. [1] However, there are many different terms, definitions, and rules concerning what operating reserves entail. In general, operating reserves are classified into three categories, regulation, spinning, and non-spinning reserves. Regulation reserves which are operating and synchronized with the SO controlled grid produce active power that can be incremented or decreased instantaneously through Automatic generation control (AGC). [14] Spinning reserves are available almost instantaneously to stabilize the system frequency upon the outage of a large generation unit in the system. Their time response varies from a few seconds to about 5 minute and requires no notification. Non-spinning reserves are similar to the spinning reserves, but it is not synchronized to the grid. Therefore, its time frame varies from several minutes to half an hour or more. [14] This classification operating reserves are applicable to the North American Electric Reliability Corporation (NERC). NERC classify operating reserves as follows: allocated for nonevents which is normal operation dealing with deviations from schedules caused by forecast errors and variability inside a scheduling period, corresponding to regulation, and reaction to rarer events, such as those caused by contingency events, analogous with spinning and non-spinning reserves. [15] However, in Europe, UCTE defines operating reserves in three categories: primary, secondary and tertiary control reserves. $[2,13]$ This approach address operating reserves services from the point of view of their time of deployment following the occurrence of a contingency as well as the corresponding control strategies behind their deployment. [11] During the primary regulation interval, generators automatically adjust to disturbances as their speed governors respond to deviations from the nominal system frequency through their droop characteristics. [13] In the secondary regulation called AGC, generators automatically vary their generation set points proportionally to some participation factors with the goal of nullifying the area control error in following normal load variations. Finally, during the tertiary regulation interval, typically with time constants exceeding $10 \mathrm{~min}$, a variation of the predisturbance set points is scheduled for each credible contingency within the prescribed generation, demand, and transmission flow limits, while ensuring that the area control error is zero and that power balances at every bus. [7] As a result, there are various methods were applied to classify the operating reserves. It is difficult to determine that which methods are better for applying to manage the operating reserves. Therefore, in this paper, we adopt UCTE classification method which is divided into primary, secondary, and tertiary reserves. In addition, we propose the DLC resource in the operating reserves.

\section{Required New Approach to Determine the Amount Operating Reserve Capacity}

In the areas of UCTE, the existing method to determine the amount of operating reserve capacity divided into three categories, primary, secondary, and tertiary control reserves has been to use practical experience. The practical experience may set operating reserve requirement by establishing that the amount of generation reserve available should be at least greater than or equal to the largest generation unit committed and should be no less than a certain percentage of total operating capacity. [1] As a result, the existing rule for determining reserve requirements does not consider technical and economic aspects. In addition, this approach is also not suitable in terms of power system reliability when the large load fluctuation occurs. Therefore, this section presents the need for a new calculation method to determine reserve requirements.

\subsection{Abnormal temperature}

Many events can cause power system imbalance between demand and supply. Among the events, SO are becoming concerned about maintaining reliable and secure power system operation due to the increased load fluctuations because of abnormal temperature. Even though generator and network outage did not occur, operating reserves can 
be scarce due to the large load volatility. A typical example is 9.15 South Korea rolling black-out occurred on September 15, 2011. Korea power exchange (KPX) forecast 6,400thousand $\mathrm{KW}$ as the peak load. However, the real peak demand is over the 6,700thousand $\mathrm{KW}$ because abnormal hot weather lead to an increase in electricity demand such as air-conditioner and the remaining operating reserves were deficient to make up imbalance between demand and supply. Finally, KPX carried out rolling black-out to prevent collapse of the power system. KPX did not consider the demand volatility in real time when they determine the operating reserve requirements. As demand volatility is random value, it is difficult to apply to evaluate the amount of operating reserves accurately. However, the uncertainties would decrease when determining the operating reserve requirements lead time is shortened. Therefore, when we determine the operating reserve requirements in real time, power system can be operated to withstand large load fluctuations.

\subsection{Renewable energy resources}

The need to reduce pollutant gas emissions has led to a large scale development of distributed renewable energy generators in future power system. Distributed generator, such as photovoltaic or wind power generators, are used to reduce fuel consumption and greenhouse gas emissions. However, the output power fluctuation of renewable resources may cause excess fluctuations of the demand due to the variation of wind speed and sunshine intensity. [6, 7] Therefore, power systems with high distributed generator penetration experience increased variability and uncertainty, such that new method to determine the required operating reserve is needed.

\section{Mathematical Modeling}

The amount of operating reserve which is needed at any time is a dynamic problem and the required reserve capacity is necessary to be adjusted over short time period that is reflecting the real time load imbalance. Therefore, in this paper, we determine the secondary, tertiary and DLC reserve capacity during short time period. At first, the formulation of this problem is shown and then we introduce the load imbalance equation and reserve market model. Next, we represent how to operate each reserve resource that is secondary, tertiary, DLC and load shedding. Finally, PDP is provided to solve this problem.

\subsection{Problem formulation}

The outline of determining capacity of real-time reserve is sketched in Fig. 1. The cost for compensating the load imbalance is divided as capacity cost and energy cost. Because the load imbalance is probabilistic information,

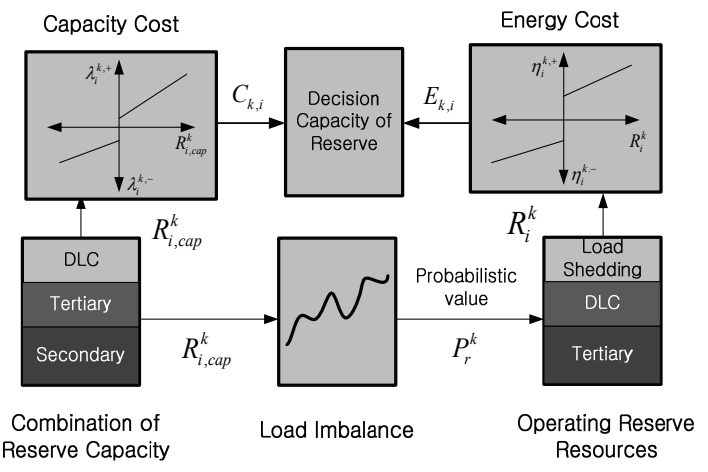

Fig. 1. Outline of determining capacity of real-time reserve

total costs are expectation value. Therefore, the objective function is to minimize the overall expectation cost in period.

$$
\min _{R_{i, c a p}^{k}, R_{i}^{k}} E_{P_{r}^{k}}\left\{\sum_{i=2}^{4} C_{k, i}+\sum_{i=3}^{5} E_{k, i}\right\}
$$

In the Eq. (1), the first term represents the capacity cost from the reserve market. In addition, the second term means the expected energy cost. The elementary of the objective function represent in the next section.

\subsection{Load imbalance equation and reserve market model}

In this section, we describe a load imbalance equation and reserve market model. At first, we consider the load demand that is composed to scheduled power supply and load imbalance in $\mathrm{k}$ period through the following equation:

$$
P_{d}^{k}=P_{s c h}^{k}+P_{r}^{k}
$$

In Eq. (2), the scheduled power supply is given value in this paper and load imbalance is related to the frequency deviations because an isolated power system is sensitive in responding to frequency deviations as follows:

$$
P_{r}^{k}=-K \int B_{f} \cdot\left(f_{k}-f_{r e f}\right)
$$

The amount of load imbalance is assumed to discrete states each state has its transition probability. The state of the load imbalance model is shown in the Fig. 2.

One of state of $\mathrm{k}$ stage varies from -3 to 3 times of the variance of load volatility based on their state position. 3 times of the variance means that the modeling of load imbalance covers $99.74 \%$ of variance. Moreover, varied state of $\mathrm{k}+1$ stage has state transition probability (i.e., $\operatorname{prb}\left(w_{v, k+1} \mid w_{j, k}\right)$ ) that is determined by applying the normal distribution function through the Fig. 2. $\operatorname{prb}\left(w_{v, k+1} \mid w_{j, k}\right)$ means the transition probability that next period's state of load imbalance will be $w_{v, k+1}$, given 

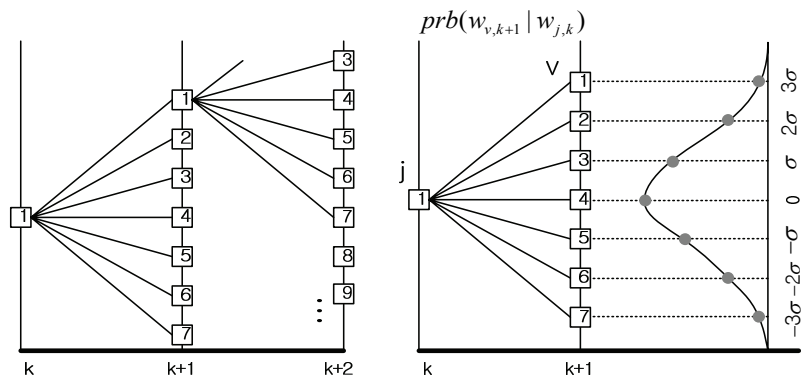

Fig. 2. Load imbalance model

that the current state is $w_{j, k}$.

The amount of load imbalance is handled by a balancing mechanism that ISO determine reserve capacity and operation. Prior to determining the reserve capacity and operation, we define the reserve market model for calculating the capacity and energy cost. We assume that the reserve market has characteristic of linear cost function as follows:

$$
\begin{aligned}
& C_{k, i}=\lambda_{i}^{k,+}+\lambda_{i}^{k,-}, \forall i=2,3,4 \\
& \lambda_{i}^{k,+}=a_{i}^{k,+} \cdot R_{i, k}^{c a p}+b_{i}^{k,+} \\
& \lambda_{i}^{k,-}=a_{i}^{k,-} \cdot R_{i, k}^{c a p}+b_{i}^{k,-}
\end{aligned}
$$

The Eq. (4) represents the capacity cost function and Eq. (5) illustrates the energy cost function. In Eq. (4), the load shedding does not account to capacity cost function because load shedding will selectively disconnect some loads not voluntarily determined in order to guarantee the system security if there is not enough the amount of remain reserve. Therefore, load shedding should be compensated for the cost of energy through the following Eq. (5).

Adjustment to the energy cost of secondary reserve does not have to do because the amount of secondary reserve is standard indicators for determining the execution time of other reserves, tertiary, DLC and load shedding. As a result, tertiary, DLC and load shedding except secondary reserve are paid for the cost of energy as shown in Eq. (5).

$$
\begin{aligned}
& E_{k, i}=\eta_{i}^{k,+}+\eta_{i}^{k,-}, \forall i=3,4,5 \\
& \eta_{i}^{k,+}=c_{i}^{k,+} \cdot R_{i}^{k,+}+d_{i}^{k,+} \\
& \eta_{i}^{k,-}=c_{i}^{k,-} \cdot R_{i}^{k,-}+d_{i}^{k,-}
\end{aligned}
$$

\subsection{How to operate reserve resources}

In this section, the way of operating reserve resources are introduced. Prior to the explanation, operating reserve resources is restricted to the following assumptions:

- When load imbalance excess the amount of secondary reserve,

1. The cost of reserve resources are committed in order:

- Committed sequence is tertiary, DLC and load shedding.

2. The amount of standard activated reserve is capacity of secondary reserve
- When the amount of secondary reserve takes all of its capacity for making up for load imbalance, tertiary reserve goes into reserve resources as much as the capacity of secondary reserve. As a result, the state of secondary reserve goes to initial position ' 0 '. Likewise, DLC and load shedding are applied in the same way.

In Eq. (3), we already defined load imbalance model during $\mathrm{k}$ period. To make up for load imbalance, reserve resources are used and corresponding equation is shown as in Eq. (6).

$$
P_{r}^{k}=R_{2, l o c}^{k+1}-R_{2, l o c}^{k}+\sum_{i=3}^{5} P_{i}^{k}
$$

Load imbalance is composed of the state of secondary reserve and activated reserve resources through the $\mathrm{k}$ period. Now then this we define each activated reserve resource that is related to the capacity of secondary reserve as following Eq. (7).

$$
\begin{gathered}
R_{i}^{k}=R_{2, k}^{c a p} \cdot n_{i}^{k} \cdot\left(1-m^{k}\right) \\
\sum_{i=3}^{5} n_{i}^{k}=\left\lfloor\left(P_{r}^{k}+R_{2, l o c}^{k}\right) / R_{2, k}^{c a p}\right\rfloor \\
n_{i}^{k}=\left\lfloor R_{i, k}^{c a p} / R_{2, k}^{c a p}\right\rfloor, \forall i=3,4,5 \\
m^{k}=\left\{\begin{array}{cc}
0, & P_{r}^{k}+R_{2, l o c}^{k}>R_{2, k}^{c a p} \\
1, & \text { otherwise }
\end{array}\right.
\end{gathered}
$$

For calculating the amount of each activated reserve resource, at first we have to define the total number of activated reserve resource as shown in Eq. (8). And then, each reserve resource is committed in order as represented in Eq. (9). In addition, we also define indicator variable. When the load imbalance does not excess the capacity of secondary reserve, it is not necessary to activate other reserve resources through the following Eq. (10). Then, the state of secondary reserve is calculated as the Eq. (11).

$$
R_{2, l o c}^{k}=\left(P_{r}^{k} \cdot m^{k}\right)+\left(P_{r}^{k}-\sum_{i=3}^{5} P_{i}^{k}\right) \cdot\left(1-m^{k}\right)
$$

Through the Eq. (7), it is possible to calculate the use of energy cost during $\mathrm{k}$ period and it reflects the cost of objective function which is to minimize the overall cost in period. In addition, as shown in equation (11), the state of secondary reserve applies to the state of PDP that is optimization method to determine the capacity of reserve resources in this paper.

\subsection{Probabilistic dynamic programming}

The formulated problem can be solved by the 


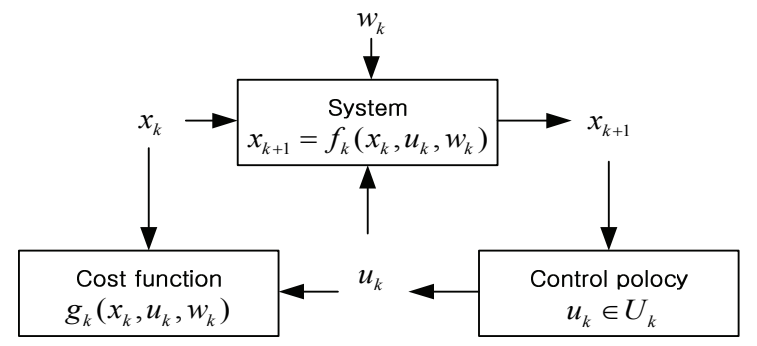

Fig. 3. PDP framework for discrete finite state problem

Probabilistic Dynamic Programming (PDP) method. The load imbalance is discretized to for the PDP framework for discrete finite state problems. [12] Then, the PDP backward algorithm is applied to determine for the optimal amount of reserve resources in period. This is our proposed scheme and the PDP framework is shown in Fig. 3.

In PDP framework, let us define:

1. State variation be the position of secondary reserve at the end of period $\mathrm{k}$.

2. Control variable be the reserve capacity and the amount of activated reserve in period $\mathrm{k}$.

3. Random variable be the uncertainty of load imbalance in period $\mathrm{k}$.

The state transition function of PDP framework can be written as:

$$
x_{k+1}=x_{k}+P_{r}^{k}-\sum_{i=3}^{5} R_{i}^{k}
$$

By substituting the Eq. (7), the state transition function is rewritten as:

$$
x_{k+1}=x_{k}+P_{r}^{k}-\sum_{i=3}^{5} R_{2, k}^{c a p} \cdot n_{i}^{k} \cdot\left(1-m^{k}\right)
$$

The controller in PDP framework is shown as:

$$
u_{k}=\mu_{k}\left(P_{i, k}^{c a p}\right), \forall i=2,3,4
$$

The cost function in PDP framework is defined as:

$$
\begin{aligned}
& g_{k}\left(x_{k}, u_{k}, w_{k}\right)=C_{k, i}+E_{k, i} \\
& C_{k, i}: \forall i=2,3,4, E_{k, i}: \forall i=3,4,5
\end{aligned}
$$

Then, the cost-to-go function is calculated as:

$$
\begin{aligned}
& V_{N}\left(x_{N}\right)=V_{1}\left(x_{1}\right)=0 \\
& V_{k}\left(x_{k}\right)=\min _{u_{k}}\left\{\sum_{j} \operatorname{prb}\left(w_{v, k+1} \mid w_{j, k}, u_{k}\right)\right. \\
& \left.\cdot\left[g_{k}\left(x_{k}, u_{k}, w_{k}\right)+V_{k+1}\left(x_{k+1}\right)\right]\right\} \\
& k=1,2, \ldots, N-1
\end{aligned}
$$

The load imbalance $w_{k}$ indicates the random variable in PDP problem. Therefore, the cost-to-go function has expectation value as shown in Eq. (16). In this equation, $V_{k}\left(x_{k}\right)$ is the minimum expected reward that can be earned during stages $\mathrm{k}, \mathrm{k}+1, \ldots$, end of this problem.

In addition, $\operatorname{prb}\left(w_{v, k+1} \mid w_{j, k}, u_{k}\right)$ indicates the probability that next period's load imbalance state will be $w_{v, k+1}$, given that the state of current stage is $w_{j, k}$ and the action $u_{k}$ is chosen. As a result, through the Eq. (16), optimal amount of reserve resources is determined at the state of load imbalance in all stages.

\section{Case study}

In this section, the proposed determining the amount of reserve resources method is applied to a study case. We simulate the time basis of 15 minutes in reserve market, $\mathrm{N}=60 / 15+1=5$ periods in an hour. Therefore, proposed method in this paper determines the capacity of reserve resources every 15 minutes.

The reserve market cost data is assumed through the following Tables (1) and (2). It represents the capacity and energy cost coefficient respectively. Generally, reserve resource of the highest capacity cost is secondary reserve and the lowest one is DLC reserve. However, reserve resource of the highest energy cost is load shedding reserve and the lowest one is tertiary reserve.

The load imbalance uncertainty is modeled through standard normal distribution. One of random state of load imbalance could be moved to state of 7 cases at the end of next stage. Table 3 indicates the state transition probability.

Fig. (4) illustrates the load imbalance model and the Table (4) indicates the number of states in each stage. The bold red lines means one of the path of load imbalance and the required reserve capacity at each state of load

Table 1. Data of capacity cost coefficient

\begin{tabular}{c|c|c|c}
\hline $\begin{array}{c}\text { Capacity cost } \\
\text { coefficient }\end{array}$ & $\begin{array}{c}\text { Secondary } \\
\text { reserve }\end{array}$ & $\begin{array}{c}\text { Tertiary } \\
\text { reserve }\end{array}$ & DLC \\
\hline$a_{i}^{k,+}$ & 100 & 10 & 2 \\
$b_{i}^{k,+}$ & 15 & 10 & 1 \\
$a_{i}^{k,-}$ & 10 & 1 & 0.5 \\
$b_{i}^{k,-}$ & 1 & 1 & 0.1 \\
\hline
\end{tabular}

Table 2. Data of capacity cost coefficient

\begin{tabular}{c|c|c|c}
\hline $\begin{array}{c}\text { Capacity cost } \\
\text { coefficient }\end{array}$ & $\begin{array}{c}\text { Secondary } \\
\text { reserve }\end{array}$ & $\begin{array}{c}\text { Tertiary } \\
\text { reserve }\end{array}$ & DLC \\
\hline$c_{i}^{k,+}$ & 100 & 200 & 1000 \\
$d_{i}^{k,+}$ & 10 & 15 & 100 \\
$c_{i}^{k,-}$ & 1 & 5 & 10 \\
$d_{i}^{k,-}$ & 20 & 40 & 100 \\
\hline
\end{tabular}




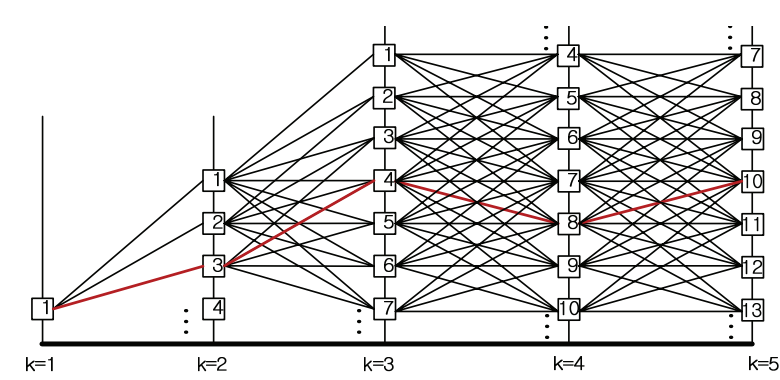

Fig. 4. The state of load imbalance in 5 stages

Table 3. Data of state transition probability

\begin{tabular}{c|c|c}
\hline state of k stage & State of $\mathrm{k}+1$ stage & State transition probability \\
\hline & $w_{v, k+1}=w_{j, k}+3 \sigma$ & 0.0109 \\
& $w_{v, k+1}=w_{j, k}+2 \sigma$ & 0.0546 \\
$w_{v, k+1}=w_{j, k}+\sigma$ & 0.1598 \\
$w_{j, k}$ & $w_{v, k+1}=w_{j, k}$ & 0.5467 \\
& $w_{v, k+1}=w_{j, k}-\sigma$ & 0.1598 \\
& $w_{v, k+1}=w_{j, k}-2 \sigma$ & 0.0546 \\
& $w_{v, k+1}=w_{j, k}-3 \sigma$ & 0.0109 \\
\hline
\end{tabular}

Table 4. Number of state in each stage

\begin{tabular}{c|c|c|c|c|c}
\hline Stage & $\mathrm{k}=1$ & $\mathrm{k}=2$ & $\mathrm{k}=3$ & $\mathrm{k}=4$ & $\mathrm{k}=5$ \\
\hline Number of state & 1 & 7 & 13 & 19 & 25 \\
\hline
\end{tabular}

Table 5. Optimal necessary reserve capacity

\begin{tabular}{c|c|c|c|c|c}
\hline Stage & $\mathrm{k}=1$ & $\mathrm{k}=2$ & $\mathrm{k}=3$ & $\mathrm{k}=4$ & Fixed value \\
\hline State Index & 1 & 3 & 4 & 8 & \\
\hline $\begin{array}{c}\text { Capacity of } \\
\text { Secondary reserve }\end{array}$ & 1 & 1 & 1 & 1 & 2 \\
\hline $\begin{array}{c}\text { Capacity of Tertiary } \\
\text { reserve }\end{array}$ & 2 & 3 & 5 & 4 & 8 \\
\hline Capacity of DLC & 1 & 1 & 1 & 1 & 0 \\
\hline
\end{tabular}

Table 6. Comparing the cost function

\begin{tabular}{c|c|c}
\hline & Case 1 & Case 2 \\
\hline Total $\operatorname{cost}(\$)$ & 15918 & 12249 \\
\hline
\end{tabular}

imbalance is informed through the Table 5 .

Now, we divide two cases for comparing the cost function when the load imbalance occurs as bold red lines in Fig. (4). In case 1, the optimal required reserve capacity applies fixed value during the all periods without consideration of real-time load imbalance. The fixed required reserve capacity is calculated as the Table (5). In case 2, optimal necessary reserve capacity is determined respectively at each period according to the load imbalance.

In the Table (6), proposed method to determine required reserve capacity, the case 2 , is more effective than the case 1 because it is possible to cope with load imbalance. For example, in case 1, when the amount of load imbalance is small during a certain period of time, surplus amount of reserve capacity would be increased. Therefore, it is reasonable that SO determine the reserve capacity every
15 minutes according to the state of load imbalance information by using the proposed method.

\section{Conclusion}

The characteristic of future power system is that the uncertainty of load variation is getting greater because smart-grid with high renewable resources penetration and abnormal temperature induce demand to increased variability and uncertainty. Therefore, the operation of reserve is an important component of power system.

This paper provides a framework for determining the optimal required reserve capacity. This problem formulation is then put into a Probabilistic Dynamic Programming (PDP) framework where the PDP backward algorithm can be applied. Therefore, the main contribution of this paper is to present an optimization model that is PDP to determine the amount of reserve capacity taking into account the real time load imbalance. In the case study, we apply two cases to operation of reserve. In case 1, real time load imbalance is not considered while in case 2, the proposed scheme is adopted. The decrease in the cost of case 2 compared to case 1 has shown the effectiveness of our proposed scheme.

\section{Nomenclature}

$\mathrm{k} \quad$ The time index of real-time market (15 minutes)

$\mathrm{i} \quad \mathrm{i}=2$ : Secondary reserve index

$i=3 \quad$ Tertiary reserve index

$i=4 \quad$ DLC reserve index

$\mathrm{i}=5 \quad$ Load shedding reserve index

$C_{k, i} \quad$ Capacity cost, [\$/MW]

$E_{k, i} \quad$ Energy cost, [\$/MWh]

$P_{d}^{k} \quad$ Demand at k period, [MW]

$P_{s c h}^{k} \quad$ Scheduled generation at k period, [MW]

$P_{r}^{k} \quad$ Load imbalance at k period, [MW]

$\mathrm{K} \quad$ Gain of integrator

$B_{f} \quad$ Frequency bias constant

$f_{k} \quad$ Frequency value at $\mathrm{k}$ period, $[\mathrm{Hz}]$

$f_{\text {ref }} \quad$ Reference frequency $(60 \mathrm{~Hz})$

$a_{i}^{k,+}, a_{i}^{k,-}$ Gradient of positive / negative capacity cost function

$b_{i}^{k,+}, b_{i}^{k,-}$ y-intercept of positive / negative capacity cost function

$c_{i}^{k,+}, c_{i}^{k,-}$ Gradient of positive / negative energy cost function

$d_{i}^{k,+}, d_{i}^{k,-}$ y-intercept of positive / negative energy cost function

$R_{i, k}^{c a p} \quad$ The amount of capacity [MW]

$R_{i}^{k} \quad$ The amount of use of energy [MWh]

$R_{2, l o c}^{k} \quad$ The location of secondary reserve [MW]

$n_{i}^{k} \quad$ The number of activated reserve resource

$m_{i}^{k} \quad$ Indicator variable to whether or not to activated reserve resources 
$x_{k} \quad$ State variable in PDP framework

$u_{k} \quad$ Control variable in PDP framework

$w_{k} \quad$ Random variable in PDP framework

$g_{k}\left(x_{k}, u_{k}, w_{k}\right)$ Cost function in PDP framework

$f_{k}\left(x_{k}, u_{k}, w_{k}\right)$ State transition function in PDP framework

$V_{k}\left(x_{k}\right) \quad$ Cost-to-go function in DP framework

$x_{0} \quad$ Initial state

$x_{n} \quad$ Ending state

\section{References}

[1] Jianxue Wang, Xifan Wang, Yang Wu, Operating reserve model in the power market. IEEE Trans. Power Syst. 2005, 20, 223-229.

[2] UCTE Operation Handbook-Policy 1-Load-Frequency Control and Performance, Prelim vI.2, 3/10/2002.

[3] K. W. Cheung, P. Shamsollahi, D. Sun, J. Milligan, and M. Potishnak, Energy and ancillary service dispatch for the interim ISO New England electricity market, IEEE Trans. Power Syst. 2000, 15, 968-974.

[4] Soler, D.; Frias, P., Gomez, T., Platero, C.A., Calculation of the elastic demand curve for a dayahead secondary reserve market. , IEEE Trans. Power Syst. 2010, 25, 615-623.

[5] E. Peeters, R. Belhomme, C. Batlle, F. Bouffard, S. Karkkainen, and D. Six, ADDRESS: Scenarios and architecture for active demand development in the smart grids of the future, presented at the 20th Int. Conf. Exhibition Electricity Distribution (CIRED), Prague, Czech Republic, Jun. 8-11, 2009. Paper 0406.

[6] Holttinen, H., Milligan, M., Ela, E., Menemenlis, N., Methodologies to determine operating reserves due to increased wind power. Power and Energy Society General Meeting, 2013 IEEE, 1-10.

[7] Enrique Bustamante-Cedeno, Sant Arora, Sensitivity of generation reserve requirements in deregulated power systems. Electric Power Systems Research 2008, 78, 1946-1952.

[8] Jianming Chen, Lee, F. N., Breipohi, A. M., Adapa, R., Scheduling direct load control to minimize system operation cost. IEEE Trans. Power Syst. 1995, 10, 1994-2001.

[9] Wen-Chen Chu, Bin-Kwie Chen, Chun-uei Fu, Scheduling of direct load control to minimize load reduction for a utility suffering from generation shortage. IEEE Trans. Power Syst. 1993, 8, 15251530 .

[10] Riberio, P. F., Johnson, B. K., Crow, M. L., Arsoy, A., Liu, Y., Energy storage systems for advanced power applications. IEEE Trans. Power Syst. 2001, 89, 1744-1756.

[11] Yuen, C., Oudalov, A., Timbus, A., The provision of frequency control reserves from multiple microgrids. IEEE Trans. Industrial Electronics. 2011, 58, 173-183.

[12] Bertsekas, D.P. Dynamic Programming and Optimal
Control; Athena Scientific: Belmont, MA, USA, 1995.

[13] Galiana, F.D., Bouffard, F., Arroyo, J.M., Restrepo, J.F., Scheduling and pricing of coupled energy and primary, secondary, and tertiary reserves. Proceedings of the IEEE 2005, 93, 1970-1983.

[14] J. Aghaeia, H. Shayanfara, N. Amjadyb, Multiobjective market clearing of joint energy and reserves auctions ensuring power system security. Energy Conversion and Management 2009, 50, 899-906.

[15] R. J. Koessler, J. W. Feltes, and J. R. Willis, A methodology for management of spinning reserve requirements, in Proc. IEEE Power Eng. Soc. Winter Meeting, 1999, vol. 1, pp. 584-589

[16] Leite da Silva, A.M., Alvarez, G.P., Operating reserve capacity requirements and pricing in deregulated markets using probabilistic techniques. Generation, Transmission \& Distribution, IET 2007, 1, 439-446.

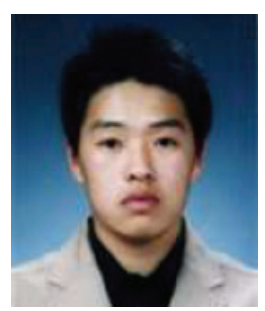

Joon Hyung Park was born in Korea on 1982. He received B.S.E.E degree in Electrical Engineering from KyungHee University, Suwon, Korea in 2007. Currrently he is working on Ph.D course in the Department of Electrical Engineering a Seoul National University. His present research interests are Generation expansion plan and voltage stability. Power System Automation Control.

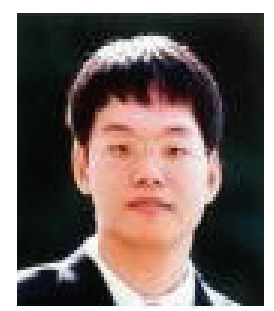

Sun Kyo Kim was born in Seoul, Korea on 1981. He received B.S.E.E. degree in Electrical Engineering from Hanyang University, Seoul, Korea in 2006 and the Ph.D. from Seoul National University, Seoul Korea, in 2014. Currently he is a senior re-searcher in the power industry research team at KEPCO Economy \& Management Research Institute. His present research interests include ancillary service market design, real-time market operation, and frequency control.

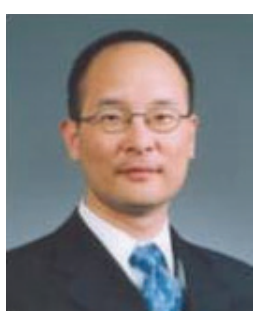

Yong Tae Yoon (M'04) received B.S., M. Eng., and Ph.D. degrees from M.I.T., Massachusetts, USA, in 1995, 1997, and 2001, respectively. Currently, he is an Associate Professor in the School of Electrical Engineering and Computer Science at Seoul National University, Seoul, Korea. His research interests include electric power network economics, power system reliability, and the incentive regulation of independent transmission companies. 